\section{Derribando muros: un aporte desde la extensión universitaria a los procesos de sustitución del manicomio}

Mariana Chidichimo

Facultad de Psicología de la

Universidad Nacional de Rosario,

Argentina.

marianachidichimo@hotmail.com
A 100 años de la Reforma Universitaria de 1918 /

Intervenciones

RECEPCIÓN: 20/06/17

ACEPTACIÓN FINAL: 24/10/17

\section{Resumen}

Se reflexiona sobre una experiencia de construcción de nuevas formas de intervención en salud mental. La Ley Nacional de Salud Mental y Adicciones № 26657 (2010-2013) propone la sustitución y cierre definitivo de los hospitales psiquiátricos y la construcción de un nuevo modelo de atención.

La Comisión Nacional Interministerial en Políticas de Salud Mental y Adicciones recomienda a las universidades incorporar en sus planes de estudios el enfoque de derechos humanos, la interdisciplina, la salud pública y la inclusión social como ejes transversales para la formación, extensión e investigación.

En el año 2013 comienza a funcionar en la ciudad de Rosario el proyecto de extensión universitaria "Derribando Muros: cine-debate los domingos en el

Gomecito", espacio de práctica interdisciplinaria y participativa en consonancia con el paradigma que la legislación actual propone. Se reflexiona sobre esta intervención comunitaria de promoción de la salud mental y de generación de lazo social que se propone desnaturalizar las significaciones imaginarias que la sociedad tiene respecto de la locura y su tratamiento.

\section{Palabras clave}

- Extensión universitaria

- Usuarios de servicios de salud mental

- Sustitución del manicomio

- Inclusión social

\section{Resumo}

Reflete-se em torno a uma experiência de construção de novas formas de intervenção em saúde mental.A Lei Nacional de Saúde e Adicções N 26657 (2010-2013) propõe a sistituição e fechamento definitivo de hospitais psiquiátricos e a construção de um novo modelo de atenção.

A Comissão Nacional Interministerial em Políticas de Saúde Mental y Adicções recomenda às Universidades a incorporar em seus currículos a abordagem dos direitos humanos, a interdisciplinaridade, a saúde pública e a inclusão social como pilares para a formação, extensão e pesquisa.

Em 2013 começa a funcionar na cidade de Rosario o projeto de extensão universitária Derribando Muros: cinema-debate nos domingos no Gomecito, espaço de prática interdisciplinar e participativo de acordo com o paradigma que a legislação atual propõe. Reflete-se em torno a essa intervenção comunitária de promoção da saúde mental e de geração de laço social que propõe-se desnaturar as significações imaginárias que a sociedade tem sobre a loucura e seu tratamento.

Palavras-chave

- Extensão Universitária

- Usuários de serviços de saúde mental

- Sustituição do manicômio

- Inclusão social
Para citación de este artículo

Chidichimo, M. (2017). Derribando muros: un aporte desde la extensión universitaria a los procesos de sustitución del manicomio. Revista +E versión en línea, 7(7), 330-337. Santa Fe, Argentina: Ediciones UNL. 


\section{Algunas líneas para historizar el problema}

Desde la existencia nómade y errante del loco dejado fuera de los muros de las ciudades, pasando por el hospicio y su función de hospedaje de los marginales, aquellos que simbolizan una amenaza al orden social, hasta su caracterización como representante del peligro social y de la enfermedad mental, con la institucionalización de la locura que ordena la medicina, se asiste a una historia de encierro y exclusión de lo diferente. Cada sociedad y cada época han construido los problemas en este terreno, así como las respuestas a los mismos, las que se concretizan en saberes que legitiman prácticas y se plasman en políticas públicas.

El campo de la salud mental en Argentina, debido a la conformación cultural e histórica del país, ha sido influenciado por distintas experiencias de reforma psiquiátrica, pero también por los avances y retrocesos producto de las decisiones gubernamentales en políticas sanitarias. El concepto Reforma Psiquiátrica es tomado en un sentido general, tal como lo plantea Amarante (2006) refiriéndose al proceso histórico de reformulación crítica y práctica que tiene como objetivos y estrategias el cuestionamiento y elaboración de propuestas de transformación del modelo clásico y del paradigma de la psiquiatría, sin desconocer que este concepto se presenta política y conceptualmente problemático, ya que es indicativo de transformaciones superficiales, cosméticas, accesorias en oposición a transformaciones estructurales, radicales y de base. Su riqueza radica en que permite contener distintas experiencias. Hablar de reformas no implica connotarlas positivamente a priori, ni hablar de la superación del paradigma fundante de la intervención psiquiátrica (Alberdi, 2003). Hay que destacar que hacia fines de la década del '50 comenzó a consolidarse el campo de la salud mental en nuestro país y se desarrollaron múltiples e innovadoras experiencias que, fragmentarias y ampliamente resistidas, convivieron con la estructura manicomial. Durante la década del ' 60 el psicoanálisis tuvo una fuerte impronta en las diversas experiencias que conformaron el campo y las reformas en salud mental. $Y$ en consonancia con las reformas que se daban en el mundo, se instalaron nuevos dispositivos de formación y atención (las primeras residencias de salud mental, salas de internación en hospitales generales, hospitales de día y comunidades terapéuticas) $y$, a la vez, se difundieron los tratamientos grupales, institucionales y familiares que mostraban la potencialidad de extender los límites del psicoanálisis.

Si a principios de la década del '70 se había perdido la apuesta de liquidar al poder manicomial, durante la dictadura del '76 se produjo la demolición de todas las experiencias progresistas en salud mental. En 1983, con la recuperación democrática, se gestaron experiencias promovidas en casi todo el país por la recién creada Dirección Nacional de Salud Mental. En cambio, las políticas neoliberales implementadas durante los '90 funcionaron como límite para el avance de las reformas, aunque la organización alcanzada durante el período anterior posibilitó que algunas provincias sostuvieran sus experiencias de reformas y se comenzaran a generar legislaciones específicas en salud mental:

"En este sentido, la historia de las alternativas al poder psiquiátrico en el país es la historia de las experiencias piloto ya que nunca, durante el transcurso del siglo XX, hubo una decisión política desde el Estado que organizara de manera diferente la Salud Mental" (Carpintero y Vainer, 2005:191).

En el nuevo milenio resurgen proyectos fuertemente vinculados a la defensa de los derechos humanos y la inclusión de nuevos sujetos de Derecho y de nuevos derechos para las personas con padecimiento subjetivo. La visibilización de la segregación y maltrato al que son sometidas las personas con padecimiento subjetivo en las instituciones manicomiales y la lucha generada desde diversas experiencias organizativas locales impulsan la sanción y posterior reglamentación de la Ley Nacional de Salud Mental y Adicciones Nº 26657 (2010-2013) (en adelante LNSMyA). Por primera vez en la historia del país se cuenta con una legislación nacional que propone la sustitución y cierre definitivo de los hospitales psiquiátricos, públicos y privados. A su vez, esta ley plantea la construcción de un modelo de atención en salud mental con el doble propósito de lograr la accesibilidad de la población con padecimiento subjetivo que no recibe atención y diseñar una red de dispositivos alternativos que sustituyan a las instituciones psiquiátricas y se ocupen de los problemas de la población allí alojada.

El informe del Centro de Estudios Legales y Sociales (CELS, 2008) realizado entre los años 2004 y 2007 en diferentes regiones del país pone en evidencia que la institucionalización a gran escala y los abusos que la acompañan se deben a una política que, básicamente, continúa invirtiendo en establecimientos que segregan a las personas con padecimientos subjetivos. A la vez se advierte que esta situación es injustificada en tanto Argentina dispone de recursos humanos capacitados, de modelos de reforma de salud mental largamente reconocidos, de leyes progresistas que favorecen la promoción de la atención primaria de la salud mental y de servicios ubicados en la comunidad.

En efecto, tanto la LNSMyA como los movimientos de desmanicomialización, promueven ciertos cambios políticos y asistenciales, entre los cuales se destacan como prioritarios los que plantean la necesidad de fuertes modificaciones en las instituciones, en el sentido de transformar las relaciones entre los centros asistenciales, los y las usuarias, sus familiares y la comunidad en su conjunto. Estos cambios se realizan con vistas a la consolidación gradual de formas positivas de participación tanto en los servicios como en los tratamientos, introduciendo a los y las usuarias como 
sujetos activos y protagonistas en el proceso de construcción de sus vidas, de manera de superar las formas tradicionales de contención, tutela y segregación.

La legislación actual abre una serie de posibilidades de intervención desde matrices emancipatorias que exigen la promoción de condiciones dignas de asistencia que no estén divorciadas del ejercicio de los derechos civiles, políticos y sociales, lo cual implica una revisión sustancial de la forma de entender y tratar los graves padecimientos psíquicos, especialmente las formas de asistencia más colonizadas por el modelo médico hegemónico. Entendemos que este es un momento propicio para la discusión y redefinición de servicios y prácticas en salud mental, y que uno de los desafíos actuales es intervenir en la desarticulación de la universidad con el campo de las prácticas públicas en salud mental. A este respecto, el artículo $33^{\circ}$ de la LNSMyA estipula que la autoridad de aplicación debe desarrollar recomendaciones dirigidas a las universidades públicas y privadas para que la formación de los profesionales en las disciplinas involucradas sea acorde con los principios, políticas y dispositivos que se establezcan en cumplimiento de la ley.

La CONISMA (Comisión Nacional Interministerial en Políticas de Salud Mental y Adicciones), luego de consultas con diversos actores, elaboró un documento que recomienda a las universidades públicas y privadas incorporar en sus planes de estudios cuestiones relativas al enfoque de derechos humanos, a la interdisciplina e intersectorialidad, a la salud pública y a la inclusión social como ejes transversales para la formación, extensión e investigación. Situación que obliga a pensar lineamientos que orienten la adecuación de la formación de los profesionales de las carreras de Trabajo Social, Enfermería, Medicina, Abogacía y Psicología, en principio y como mínimo.

Las modificaciones legislativas y conceptuales en torno a salud mental interrogan a la formación y capacitación de recursos humanos, no solo en los ámbitos académico-disciplinares sino también en la formación en servicio y en relación con los actores no convencionales. En la actualidad, se constata un divorcio entre la universidad y los servicios: la investigación, capacitación y evaluación son ajenas o distantes de las necesidades de las poblaciones y trabajadores, y en general desarticuladas. Un estudio realizado por el Global Forum for Health Research (GFHR) y la OMS en los países en desarrollo mostró claramente que la investigación y la atención marchan por distintos derroteros (Sharan, et al., 2007).

En este sentido, las prácticas de extensión universitaria son una herramienta adecuada y con enorme potencialidad para comenzar a desandar los efectos de aislamiento y fragmentación producidos por las políticas neoliberales en materia de educación y salud.
Si se reconoce que el tema central de la problemática de la extensión hoy es la producción de conocimiento sobre la realidad, esto implica ir más allá de situar acciones de transferencia al medio de lo producido dentro de la academia, ya que la idea de transferir algo implica una concepción del otro despojado, insuficiente, diferente, carente desde un nosotros que tiene algo para decir, aportar o enriquecer. Arribar a la idea de construcción del conocimiento entre diferentes instituciones, organizaciones y actores sociales y políticos tiene consecuencias en las formas que asumirá el diálogo con estos otros. Se constituye así una forma de relación de la universidad con su sociedad a la cual considera no solo como objeto de la acción sino como fuente de saber. Entonces, la tarea es la construcción con la comunidad de sus problemas y la implementación de estrategias colectivas y cooperativas que aporten a la resolución.

Se vuelve indispensable problematizar también las categorías de beneficiarios o destinatarios en tanto aluden a una posición pasiva de recepción incompatible con la idea de participación. Grassi (2003) puntualiza que ser beneficiario implica poseer una carencia que el Estado puede subsanar con una respuesta focal. El pasaje a sujeto de derechos convierte a las necesidades en derechos civiles, culturales, sociales, bajo el principio de universalidad. En este sentido, el término "usuario" se institucionalizó en Brasil por la legislación del Sistema Único de Salud en procura de destacar el protagonismo de los que anteriormente eran pacientes, y pasó a ser adoptado con un sentido singular en el campo de la salud mental en la medida en que significó un desplazamiento del lugar social ocupado por las personas con padecimiento psíquico y la necesidad de que las personas sean involucradas en las soluciones, derivaciones y tratamientos y evitar que sean excluidas del proceso de atención (Amarante, 2009).

El reconocimiento de la palabra de los usuarios de servicios de salud mental es una deuda histórica en la mayoría de las prácticas instituidas del sector. Este proyecto pretende estimular la inclusión de la perspectiva de usuario/as de salud mental en el desarrollo de la experiencia y en los debates sobre los procesos de reforma locales, dentro y fuera de la universidad.

La co-construcción de conocimientos con otros actores sociales involucrados en la problemática permite visibilizar la necesidad de revisar desde un posicionamiento ético, los planes de estudios y programas de las distintas carreras, así como los modos de transmisión y los roles profesionales construidos.

Una reforma sanitaria no puede ser solo de los servicios asistenciales, debe incidir en el imaginario de la población y en el de cada profesión, ya que estos son los espacios donde se construyen las modalidades de las ofertas y demandas en salud. No es posible pensar entonces una reforma sanitaria escindida de la discusión del perfil profesional y de la formación de recursos humanos, aspecto nodal en la planificación de políticas públicas. 


\section{La práctica y sus fundamentos}

En el año 2013, a partir de la iniciativa de un grupo de estudiantes y docentes de la Facultad de Psicología de la Universidad Nacional de Rosario convencido de la necesidad de proponer iniciativas desde la universidad que apunten a la creación de espacios de práctica en consonancia con el nuevo paradigma que la ley de salud mental propone, surge el proyecto "Derribando Muros: cine-debate los domingos en el Gomecito". Cabe destacar que el Centro Cultural Gomecito (en adelante C.C. Gomecito) organización coparticipante del proyecto- es en la actualidad un dispositivo cultural que está transitando un proceso de adecuación a los lineamientos de la Ley Nacional de Salud Mental. Durante varias décadas fue el espacio cultural del Centro Regional de Salud Mental Dr. Agudo Ávila, institución monovalente estatal de la ciudad de Rosario. Un porcentaje importante de los usuarios/as que concurren a las actividades que allí se realizan están o han estado hasta hace poco tiempo transitando una internación por problemas de salud mental.

La problematización, diseño, planificación y evaluación del proyecto son realizados entre distintos actores: trabajadores del C.C. Gomecito, usuarias/os de servicios de salud mental, estudiantes de distintas carreras universitarias, estudiantes de Psicología cursantes de Práctica Profesional Supervisada y docentes universitarios. Con el sostenimiento de esta práctica se pretende formalizar articulaciones con el C.C. Gomecito y otros dispositivos existentes en la ciudad y la región que se proponen construir una red sustitutiva al manicomio y aportar al fortalecimiento de la Asamblea de usuarios/as de Salud Mental por sus derechos.

La expresión "servicios sustitutivos" pasó a ser adoptada para caracterizar al conjunto de estrategias que vislumbrasen efectivamente tomar el lugar de las instituciones psiquiátricas clásicas y no ser solo paralelas, simultáneas o alternativas a las mismas, no se limita a la reorganización del modelo asistencial, sino que la transformación debe alcanzar las prácticas y concepciones sociales (Amarante, 2009).

La potencialidad del concepto radica en poner en cuestión las prácticas intra y extra muros y cuestionar los modos de reproducción de la objetivación, segregación y exclusión social en las nuevas prácticas y espacios que pretenden ser sustitutivos al manicomio. La práctica está pensada como una intervención comunitaria de promoción de la salud mental y de rehabilitación psicosocial, apuesta a desandar la cronificación institucional y a la generación de lazo social.

Se parte de la premisa, ampliamente demostrada, de que las instituciones totales (Goffman, 1970) construyen una microcultura donde se borran las diferencias entre lo público y lo privado, la temporalidad es de un presente continuo que genera la imposibilidad de proyectar e investir el futuro. Estas instituciones, a través de sus prácticas, realizan una operación de homogeneización, desconocimiento de las diferencias y negación de la singularidad; separación-exclusión del sujeto solidaria del cuidado-custodia de los cuerpos. A su vez, las internaciones prolongadas causan desarraigo y ruptura de los vínculos familiares y sociales. La cronificación, patología institucional propia de la institución manicomial, suele ser la estación terminal de este proceso tantas veces denunciado. Las consecuencias son la objetivación de los sujetos, la pérdida de identidad y un progresivo deterioro subjetivo para la vida autónoma.

Hablar de promoción de la salud mental introduce de lleno en la dimensión del cuidado. El cuidado tiene una connotación mucho más amplia e integral que la atención puesto que denota relaciones horizontales, simétricas y participativas y quita la centralidad autoasignada a las instituciones y agentes del campo de la salud, en tanto reconoce que buena parte de las acciones de salud suceden en las vidas cotidianas y en las prácticas de los conjuntos sociales Los postulados sobre salud-enfermedadcuidado llevan indefectiblemente a desplazar el eje de las prácticas de la enfermedad al sujeto y a cuestionar la práctica médica centrada en las patologías individuales. Se propone, entonces, una práctica integral que incorpora la dimensión subjetiva, histórica y social tanto en el abordaje de poblaciones como de sujetos singulares. (Stolkiner y Ardila Gómez, 2012). Ulloa (1995) relaciona la salud mental con el buen trato, nombre de la ternura en los adultos y la define en tanto producción cultural como el fundamento de todo proceder antimanicomial.

"El estado de salud de un grupo resulta de la oposición permanente que existe entre aspectos saludables y protectores que el grupo disfruta y los procesos destructivos que padece de acuerdo a su especifica forma de inserción histórica [...] los factores protectores van desde la solidaridad familiar, la solidaridad y la organización grupal y de clase, el saber, la conciencia y sobre todo el poder de movilizarlos". (Breilh, 1994:62)

Robert Castel (1991) ubica la vulnerabilidad en la conjunción de la precarización del trabajo y la fragilización de los soportes relacionales que podrían asegurar una protección. Es sabido que en el extremo del proceso se ubica la desafiliación, que no necesariamente implica la ausencia completa de vínculo sino la falta de inscripción del sujeto en estructuras dadoras de sentido, lo cual se relaciona con el debilitamiento o pérdida de regulaciones colectivas y produce un individualismo por falta de marcos y no por exceso de intereses subjetivos. Escena en exceso conocida en los procesos manicomiales y en la soledad de muchas vidas contemporáneas. Al hacer énfasis en los aspectos positivos del concepto de salud mental se descentra la enfermedad y se torna visible la necesidad de acompañar la producción de vínculos significativos. 
El desarrollo del proyecto está ligado también a la producción y transmisión de conocimientos; la práctica busca articular una intervención y un espacio de formación con fines capacitadores del oficio.

Fernando Ulloa propone diferenciar la capacitación de la formación:

"La capacitación supone siempre una conceptualización de

la práctica. La formación gira más en torno a la especulación teórica, sin considerar los métodos con que se implementa la teoría. Este tipo de formación, sin mayor asidero metodológico simultáneo, es propicio a deformaciones en la clínica, y se refleja en la tendencia a practicar teorías y no a conceptualizar prácticas desde la excelencia teórica". (1995:76)

\section{Sostenimiento de la práctica}

La propuesta concreta de intervenciónconsiste en la organización y realización de un espacio de cine-debate de frecuencia semanal, los domingos por la tarde, en el C.C. Gomecito. Es central que la actividad se realice los domingos, ya que es un día de inactividad institucional y a veces de mucha soledad para quienes habitualmente transitan este $u$ otro espacio alternativo en la semana. La convocatoria a la participación en las actividades está dirigida a usuarios y usuarias de servicios de salud mental de la ciudad de Rosario y alrededores, a familiares, vecinos y amigos, así como a estudiantes, graduados/as y docentes de distintas carreras ligadas a la salud mental. Es necesario que el espacio sea abierto y que la comunidad en general sea invitada a participar con el objetivo de no acentuar el aislamiento producido por la internación y la estigmatización aún vigente sobre las personas con padecimientos subjetivos graves. Es una tarea urgente desnaturalizar las significaciones imaginarias que la sociedad tiene respecto de la locura y su tratamiento para lo cual resulta pertinente promover espacios de encuentro, socialización e inclusión social. Tanto el individualismo como la masificación llevan a la destrucción de los lazos sociales, y la reestructuración del tejido social puede darse a través de intervenciones que resitúen las singularidades en los colectivos, a partir de la producción de espacios colectivos de inscripción social.

El cine en tanto objeto cultural es una herramienta útil para propiciar un espacio-tiempo de encuentro, donde a partir de la ficción se pueda historizar el pasado, situar el presente y proyectar un futuro. Luego de cada proyección audiovisual se invita a intercambiar opiniones, historias y vivencias.

El recurso a la historización es un modo privilegiado en el proceso de construcción de salud que al mediar entre lo colectivo y lo singular, produce efectos de subjetivación. En la singularidad de cada sujeto la historia toma la forma de relación con el otro en la que se pone en juego la filiación y la identidad (Bersanker, et al., 2000).

"Ciertamente, esta representación historiadora tiene su función necesaria en una sociedad o grupo. Repara incesantemente los desgarrones entre pasado y presente. Asegura un sentido que supera las violencias y las divisiones del tiempo. Crea un teatro de referencias y de valores comunes que garantizan al grupo una unidad y una comunicabilidad simbólica". (De Certau, 1995:56)

La apuesta es la construcción de un colectivo que funcione como marco para que cada participante pueda poner a jugar su resonancia singular y, si así lo quisiera, tomar la palabra, compartir la vivencia e ir conformando el espacio. Qué películas ver, cómo elegirlas, qué servir como merienda y quién la prepara, cómo y quiénes toman las tareas de difusión, qué consensos crear para cuidarse cada uno, entre todos y al espacio, qué otras actividades hacer, cuáles son sus derechos, son algunas de las preguntas que se presentan los domingos. La coordinación del espacio es sostenida por dos estudiantes y la tarea tiene carácter rotativo. El espacio de organización planifica y resuelve tareas operativas para concretar las actividades que se proponen y desarrollan, la necesidad de realizar acciones de propaganda y convocatoria. Al inicio de la práctica estas tareas estuvieron a cargo de los estudiantes y graduados, y luego fueron tomadas en conjunto por todos los participantes interesados. Fue importante sostener la invitación y el acompañamiento a los usuarios/as a participar del diseño de cada encuentro, instancia que se realiza una vez por semana en la Facultad de Psicología. Esto implicó para algunos conocer la Universidad, para otros pedir un permiso de salida durante una internación, una mayor apropiación del espacio, y potenció las posibilidades de que se haga lugar a los intereses, deseos y también al despliegue de las capacidades y potencialidades de cada uno, aportando a la construcción de autonomía en los sujetos.

Finalmente, el espacio de retrabajo y capacitación tiene la función de evaluar de forma regular la intervención, sistematizar y elaborar nuevos interrogantes. La posibilidad de interrogar los problemas y respuestas que se construyen en torno a la salud mental a la luz del relato de las experiencias de vida, del encuentro con las instituciones y los técnicos de salud mental, previene la rigidez y la reproducción acrítica de las prácticas.

El armado de la capacitación propuesta tiene como particularidad que sus insumos provienen del recorte de los problemas realizados en los retrabajos y de la construcción de preguntas que vaya elaborando el equipo. De este modo se intenta favorecer la apropiación de problemáticas y saberes, así como la inclusión de contenidos poco abordados durante la formación de grado. A su vez, esta modalidad de capacitación permite recortar problemas para construir líneas de investigación específicas en el marco del proyecto.

En estos espacios se está atento a tener impacto en la formación de quienes sostienen la experiencia en las currículas de las distintas 
carreras y en la comunidad universitaria en su conjunto; se pretende instalar la reflexión y análisis crítico de las prácticas e incluir en la formación problemáticas respecto de la salud mental, los derechos de los usuarios y la interdisciplina.

La capacitación es una estrategia de cambio en el proceso de inclusión de los contenidos y posicionamientos que propone la legislación actual en materia de salud mental y hace a la integralidad de las prácticas educativas, a la posibilidad de aprender de una forma distinta, aprender con otros y de otros.

Parafraseando a Ulloa (1995), se apuesta a la construcción de una comunidad clínica, por aquello de aprender clínica en común a partir de la conceptualización de las prácticas cotidianas, manera de ver, leer y procesar un campo unificador de las distintas disciplinas de quienes integran la comunidad.

El enfoque de derechos es central a las prácticas transformadoras en salud mental. Su particular desarrollo en América Latina ha llevado a que movimientos y organizaciones que tienen como ideas fuerza los derechos humanos se constituyan en nuevos actores del campo. En forma paralela, sus modos de acción han promovido la aparición de otros actores, tal el caso de las organizaciones de familiares y usuarios. Son quizás estos nuevos actores los indispensables para modificar la tendencia fuertemente corporativa de los actores tradicionales del campo y su resistencia a las transformaciones. Asimismo, el enfoque de derechos no implica solamente un nuevo ordenamiento jurídico sino que es un elemento nodal en los aspectos técnicos y de gestión y se vincula indefectiblemente con la ética en la clínica singular (Stolkiner y Ardila Gómez, 2012).

El pasaje de la psiquiatría a la salud mental supone la construcción de un nuevo sujeto de intervención en las prácticas en salud mental en correlación con la lucha específica por el reconocimiento de la ciudadanía.

El movimiento de usuarios de servicios de salud mental asume una lucha contra la segregación y la estigmatización del loco y por la recuperación de su capacidad de decidir sobre los destinos de la propia vida, en nombre de la igualdad entre los hombres y de los derechos civiles. Por otro lado, son también los movimientos de usuarios y familiares los que luchan por el reconocimiento de derechos sociales especiales tales como: a un tratamiento específico y soportes asistenciales definitivos o provisorios durante el proceso de crisis, a servicios de atención psicosocial, a servicios residenciales, a esquemas especiales de trabajo y auxilios específicos. Ciudadanía especial a ser inventada tanto en el reconocimiento de los usuarios y familiares, en la producción de servicios asistenciales, como por una ciudadanía que integre la diferencia, el conflicto y la sinrazón como propias (Vasconcelos, 2000).

Los movimientos ciudadanos y de pacientes son un importante actor en las políticas sociales, exigen el empoderamiento e implicación en la gestión de la salud mental o del padecer. Hay una amplia historia desde aquellos encuentros de expacientes de hospitales psiquiátricos en las escalinatas de la Biblioteca Pública de Nueva York, en la década del "40 del pasado siglo, de los que surgió la asociación de enfermos "No Estamos Solos" (WANA); de su conversión en verdaderos movimientos de expsiquiatrizados, en los años 70 , en coincidencia con el auge de la antipsiquiatría o en movimientos institucionalizados de incidencia mundial, como Global Iniciative o de gran implantación popular y peso político como el que se está dando en Brasil. Los movimientos de pacientes, familiares y ciudadanos han sido esenciales en todos los procesos de reforma psiquiátrica, han pasado de ser marginales a participar, o pugnar por participar, en la política de los servicios que les atienden, pero sobre todo en su lucha por la defensa y conquista de los derechos civiles y la ciudadanía plena, que constituyen la parte esencial de las barreras y la discriminación (Desviat, 2011). Es una importante contribución a los problemas y desafíos actuales de este campo que las prácticas de extensión universitaria promuevan mecanismos de escucha y participación activa de los usuarios dentro y fuera de los servicios públicos. Más aún teniendo en cuenta las limitaciones concretas existentes para llevar adelante 
los cambios en la vida cotidiana de las instituciones, que van desde ausencia de políticas públicas específicas y planificaciones estratégicas —-sostenidas en registros epidemiológicos-, condiciones presupuestarias y hasta el propio maltrato hecho cultura presente en no pocas prácticas asistenciales.

Es evidente que la complejidad de los problemas de salud mental no puede ser abordada desde una sola disciplina y que la necesidad de construir un nuevo modelo de atención y de interrogar las prácticas institucionalizadas requiere pensar con urgencia la capacitación de los recursos humanos.

En este proyecto se prioriza que el equipo de trabajo esté conformado por docentes, estudiantes y graduados de distintas carreras que abordan el campo de la salud mental (Psicología, Medicina, Musicoterapia y Trabajo Social). Se impulsa el intercambio y trabajo participativo con el fin de aportar a la formación de trabajadores con herramientas para intervenir en el marco que la Ley de Salud Mental propone. A su vez, se sostiene la necesidad de construir prácticas interdisciplinarias en el grado — sostenidas por estudiantes de distintas carreras - como un paso indispensable para arribar a la construcción de herramientas que permitan realizar abordajes interdisciplinarios e intersectoriales que rompan con el dogmatismo y pongan en tensión la hegemonía disciplinar. Stolkiner plantea que la interdisciplinariedad, en tanto posicionamiento, obliga a reconocer la incompletud de las herramientas de cada disciplina y a integrar en las prácticas saberes que no son disciplinarios, que no se estructuran como conocimientos científicos:

"la interdisciplina nace, para ser exactos, de la incontrolable indisciplina de los problemas que se nos presentan actualmente. De la dificultad de encasillarlos. Los problemas no se presentan como objetos, sino como demandas complejas y difusas que dan lugar a prácticas sociales inervadas de contradicciones e imbricadas con cuerpos conceptuales diversos". (1987:313)

Con relación al abordaje de los problemas de salud mental es preciso decir, siguiendo a Stolkiner (2005), que existen obstáculos para el desarrollo del accionar interdisciplinario: la lógica hegemónica de formación y competencia individual o de mercado entre profesiones y corporaciones, así como el trabajo enfrentado a condiciones de empleo inestables de sus miembros o a exigencias de rendimiento individualmente evaluadas son solo alguno de ellos. Cabe destacar que fomentar la inclusión de derechos de los usuarios/as de servicios de salud mental y el posicionamiento interdisciplinario en la formación de grado de las carreras directamente ligadas a ello ha sido un objetivo prioritario en el diseño, planificación y desarrollo de la experiencia.

El presente proyecto pretende impactar sobre los usuarios y usuarias de servicios de salud mental y aportar a la construcción colectiva de herramientas que permitan empoderar su autonomía en tanto elemento protector de dicha salud mental. Algunos indicadores con relación a la autonomía sobre los que se puede reflexionar son la toma de la palabra y el sostenimiento de la colaboración en el armado de las distintas actividades, la participación en las instancias de presentación y evaluación del proyecto, la posición activa en torno al propio tratamiento y el cuestionamiento de las características y modalidades de las internaciones psiquiátricas. La estrategia para vehiculizar y colectivizar los cuestionamientos y acompañar el ejercicio activo de los derechos ha sido la articulación con la Asamblea de Usuarios de servicios de salud mental, espacio de vital importancia no solo para la concreción de este objetivo sino también como elemento cuestionador de la práctica en su conjunto.

En el camino para construir una red de espacios y prácticas que se proponen ser sustitutivos del manicomio, el espacio de cinedebate se ha logrado instalar como lugar de encuentro y soporte para personas usuarias de servicios de salud mental. Un aspecto relevante respecto de la concreción de este objetivo ha sido la continuidad del proyecto en el tiempo $(2013,2014,2015,2016)$.

\section{Reflexiones finales}

"Derribando muros" es una apuesta desde la Universidad —en tanto actor social productor de pensamiento y responsable de la formación de recursos humanos- a la construcción de prácticas interdisciplinarias respetuosas de los derechos humanos, inclusivas y acordes al modelo de salud mental actual. En desacuerdo con concepciones ilusorias del conocimiento como neutral, con su consiguiente desvinculación de los procesos histórico-sociales, se sostiene la responsabilidad ética y política de cuestionar los saberes instituidos, crear condiciones para la reflexión y generar espacios para el despliegue de las distintas voces. Uno de los desafíos que enfrentan las prácticas docentes en la actualidad es en cuanto a la complejidad de crear instancias de formación interdisciplinaria desde el grado, respetuosas de las especificidades cuando las distintas unidades académicas funcionan desvinculadas entre sí. Una potencialidad importante en el marco de los procesos de institucionalización y curricularización de la práctica ha sido la inclusión de estudiantes del último año de la carrera de Psicología, quienes realizan su práctica profesional supervisada en el marco del proyecto. El desarrollo de la práctica ha mostrado la necesidad de avanzar en articulaciones formales similares con cátedras de distintas carreras afines al campo de la salud mental que incluyen algún dispositivo de acercamiento a la práctica. Articulación no limitada a la inclusión de estudiantes en el espacio específico de intervención, sino que implique compartir el diseño y conceptualización de la práctica entre los docentes y cátedras involucradas. Se torna necesario construir un entre, espacio de encuentro, discusión, problematización y producción con todos los actores en juego, ya que no habrá prácticas interdisciplinarias si no se forma en la interdisciplina. 


\section{G6}

En este proyecto se prioriza que el equipo de trabajo esté conformado por docentes, estudiantes y graduados de distintas carreras que abordan el campo de la salud mental

\section{Referencias bibliográficas}

Alberdi, J. M. (2003). Reformas y Contrarreformas: Politicas de salud mental en Argentina. Rosario: UNR Editora.

Amarante, P. (2006). Locos por la vida: La trayectoria de la reforma psiquiátrica en Brasil. Buenos Aires: Ediciones Madres de Plaza de Mayo.

(2009). Superar el manicomio: Salud mental y atención psicosocial.

Buenos Aires: Topia

Bersanker, G.; Bloj, A. M.; Grande, S.; Masueco, M. I. (2000). La historización como recurso de producción de salud en situaciones de vulnerabilidad psicosocial. Ficha de cátedra. Residencia Clínica. Facultad de Psicología, UNR. Breilh, J. (1994). Nuevos conceptos y técnicas de investigación. Guía pedagógica para un taller de metodología. Argentina: Centro de Estudios y Metodología en salud.

Castel, R. (1991). La dinámica de los procesos de marginalización: de la vulnerabilidad a la exclusión. El espacio institucional. Buenos Aires: Lugar.

Carpintero, E. y Vainer, A. (2004). Las huellas de la memoria: Psicoanálisis y Salud Mental en la Argentina de los 60 y los 70. Tomo I (1957-1969). Buenos Aires: Topia.

(2005). Las huellas de la memoria: Psicoanálisis y Salud Mental en la Argentina de los 60 y los 70. Tomo II (1970-1983). Buenos Aires: Topia. CELS (2008). Vidas arrasadas: La segregación de las personas en los asilos psiquiátricos argentinos. Argentina: Siglo XXI Editores.

Comisión Nacional Interministerial en Políticas de Salud Mental y Adicciones (2013). Recomendaciones a las universidades públicas y privadas. Articulo $33^{\circ}$ Ley Nacional N²6657.

De Certau, M. (1995). Historia y Psicoanálisis. México: Universidad Iberoamericana.

Desviat. M. (2011). Panorama internacional de la reforma psiquiátrica. Ciência \& Saúde Coletiva, 16(12), 4615-4621.

Goffman, E. (1970). Internados. Buenos Aires: Amorrortu.

Ley Nacional de Salud Mental y Adicciones № 26657. Buenos Aires, Argentina (2010-2013).

Sharan, P.; Levav, I.; Olifson, S; De Francisco, A.; Saxena, S. (2007). Research capacity for mental health in low- and middle-income countries. Results of a mapping project. Geneva: Global Forum for Health Research and the World Health Organization.

Stolkiner, A. (1987). De interdisciplinas e indisciplinas. En N. Elichiry (Comp.), El Niño y la Escuela-Reflexiones sobre lo obvio. Buenos Aires: Nueva Visión.

— (2005). Interdisciplina y salud mental. IX Jornadas Nacionales de salud mental, I Jornadas Provinciales de psicología. Salud mental y mundialización: estrategias posibles en la argentina de hoy. Posadas, Misiones.

Stolkiner, A. y Ardila Gómez, S. (2012). Conceptualizando la Salud Mental en las prácticas: consideraciones desde el pensamiento de la medicina social/salud colectiva latinoamericanas. VERTEX, Revista Argentina de Psiquiatría, XXIII, $57-67$.

Ulloa, F. (1995). Novela clínica psicoanalítica. Historial de una práctica. Buenos Aires: Paidós.

Vasconcelos, E. (2000). Saúde Mental e Serviço Social: O desafio da subjetividade a da interdisciplinaridade. São Paulo: Cortez. 